\title{
Scheimpflug Topography Oriented Adequate Repositioning of a Misaligned Free Flap after Laser in situ Keratomileusis
}

\author{
Ha Eun Sim ${ }^{a}$ Min Ji Kang ${ }^{a}$ Jee Hye Lee ${ }^{a}$ Seung Hwa Baik ${ }^{b}$ \\ Sun Young Kim ${ }^{b}$ Seong Woo Lee ${ }^{c}$ Je Hyung Hwang ${ }^{a}$ \\ aDepartment of Ophthalmology, Sanggye Paik Hospital, Inje University of Korea, College of

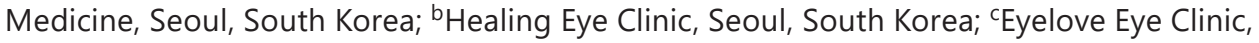 \\ Seoul, South Korea
}

\section{Keywords}

Free flap $\cdot$ LASIK $\cdot$ Repositioning $\cdot$ Scheimpflug topography

\section{Abstract}

This report describes a case of Scheimpflug topography oriented adequate repositioning of a misaligned thick free flap after laser in situ keratomileusis (LASIK). A 24-year-old patient consulted for irregular astigmatism and disoriented free right eye flap. The patient previously underwent binocular LASIK at a private clinic. During the right eye surgery, the flap was repositioned after laser ablation due to the free flap. The free flap was not repositioned to its original configuration due to insufficient preoperative corneal marking. On examination, the uncorrected visual acuity was 0.4 , and refractive power was +2.00 Dsph with -4.25 Dcyl axis 66 in the right eye. Scheimpflug topography revealed irregular right eye astigmatism. The sagittal curvature of topography showed a $40^{\circ}$ counterclockwise misalignment of the steep axis of the cornea. The free flap was repositioned by $40^{\circ}$ clockwise rotation. After this, the refractive corneal power improved to -1.00 Dsph with -1.00 Dcyl Axis 19 in the right eye. The uncorrected and best-corrected visual acuity improved to $20 / 30$ and $20 / 25$ ( $x-0.25$ Dsph -1.25 Dcyl A20), respectively. This is the first report on free flap repositioning using Scheimpflug topography. As proper flap positioning was compromised because of the free LASIK flap with no preoperative corneal marking, the flap was effectively repositioned using Scheimpflug topography. 


\section{Introduction}

A free flap develops during laser in situ keratomileusis (LASIK) operations when a corneal flap is created using a microkeratome. It is typically caused by flat cornea, poor suction, or suction loss during the operation $[1,2]$. The absence or loss of preoperative corneal markings can lead to incorrect flap orientation with serious visual consequences [3]. In this report, irregular astigmatism was corrected using Scheimpflug topography to reposition the rotationally disoriented free flap after a LASIK operation.

\section{Case Report/Case Presentation}

A 24-year-old male patient was referred to our clinic for irregular astigmatism and disoriented free flap in the right eye. The patient underwent a binocular LASIK operation at a private clinic before being referred to our clinic. A free cap was created during this operation. Prior to the operation, his best-corrected distance visual acuity was $20 / 20$ in the right eye and 20/20 in the left eye. The preoperative refractive power was $-2.25 \mathrm{Dsph}$ -0.50 Dcyl Axis 180 in the right eye and -2.25 Dsph -0.50 Dcyl Axis 10 in the left eye. The refractive corneal power was $41.00 \mathrm{D}$ in the right eye and $41.00 \mathrm{D}$ in the left eye. Meanwhile, the intraocular pressure and fundus examination were normal for both eyes. During the LASIK operation, the flap was created using a Moria M2 microkeratome (Moria, Antony, France) to the desired depth of $130 \mu \mathrm{m}$, while AMARIS 750S (Schwind, Eye-tech-solutions, $\mathrm{GmbH}$ ) laser was used for corneal ablation. A free cap was developed while creating the right eye flap. Repositioning of the free cap after corneal ablation was unsuccessful due to the lack of preoperative corneal markings. His best-corrected distance visual acuity was 20/50 (+2.00 Dsph -4.25 Dcyl axis 66) in the right eye on postoperative ocular examination. On Scheimpflug topography, the steep axis of the sagittal curvature was misaligned by approximately $40^{\circ}$ counterclockwise (Fig. 1a). Radial gentian violet marks were applied using an optical zone marker (Viscot Medical LLC, East Hanover, NJ, USA) to serve as surgical landmarks. Subsequently, the free flap was detached, and the free flap marked with radial gentian violet marks was repositioned $40^{\circ}$ clockwise, based on Scheimpflug topography. After the flap was repositioned, the uncorrected and best-corrected visual acuity improved to 20/25 and 20/20 ( $x-0.25$ Dsph -1.00 Dcyl A20), respectively. The refractive corneal power measured -1.00 Dsph-1.00 Dcyl Axis 19, and the Scheimpflug topography results showed improved alignment of the steep axis of the sagittal curvature (Fig. 1b). Additional customized photorefractive keratectomy was considered for the residual refractive error, but the patient was already satisfied with his vision. He is currently being monitored without an additional procedure.

\section{Discussion/Conclusion}

The development of a free flap during LASIK operations is an uncommon complication typically caused by flat cornea, poor suction, or loss of suction during the operation [4]. Repositioning a free flap during LASIK is simple when reference ink marks are intact [5]. The characteristic shape of a free flap would also help the surgeon to replace the flap in its original configuration. Cheng et al. [6] replaced a $3.0 \times 3.0 \mathrm{~mm}$ free flap, which was stored in balanced salt solution for $48 \mathrm{~h}$, on the stromal bed using the straight edge for alignment 4 days after initial surgery. However, in the absence of reference marks, correcting the orientation of a circular flap becomes difficult. Marks may not have been placed or may have faded during the procedure.

\section{Karger's}




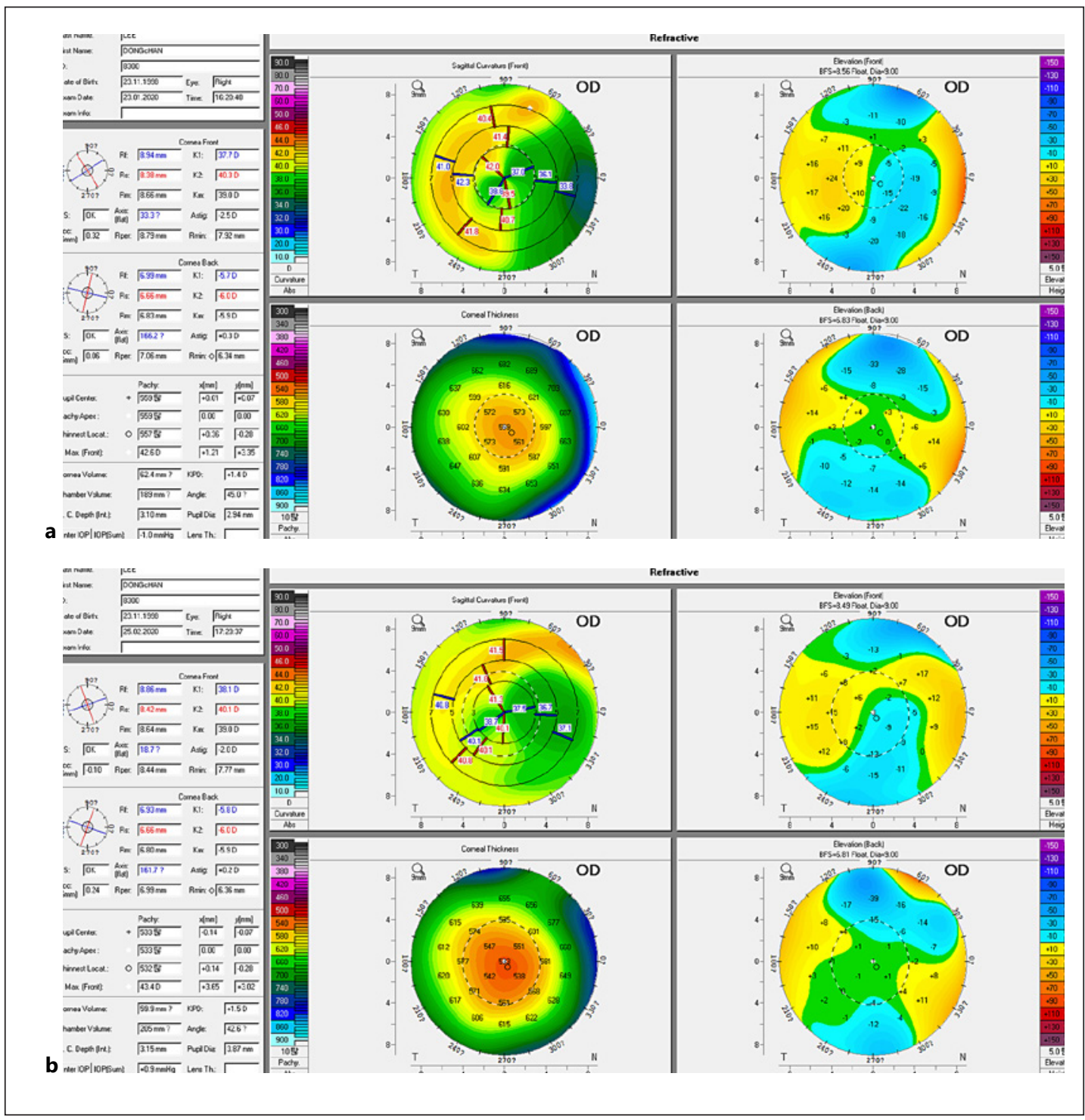

Fig. 1. Scheimpflug topography image of the patient. a Before free flap repositioning. $\mathbf{b}$ After free flap repositioning.

An incorrectly repositioned flap may lead to a severe loss of vision [1]. Nevertheless, a surgeon should not consider removing or discarding the free flap anticipating spontaneous healing analogous to surface PRK. McLeod et al. [7] reported the 2 cases of poor visual outcomes secondary to irregular astigmatism after discarding free flaps.

Theoretically, the free flap rotation would not affect the refractive power as long as the flap is perfectly round and has a uniform thickness [1]. As the thickness of a free flap is not uniform, a flap with thinner edges takes on the shape of a plus power cylindrical lens, while a flap with thicker edges takes on the shape of a minus power cylindrical lens [1]. If the cylindrical corneal cap is repositioned to the original configuration, then a refractive error does not occur. However, it is difficult to reposition the free cap to the initial configuration without preoperative markings. Todani et al. [2] reported that the free flap created using a Moria M2 microkeratome can be repositioned to the original configuration using the dot method. In our case, however, the free flap was unsuccessfully repositioned to the initial configuration as the 
dot method was not used. Hovanesian and Maloney [1] developed the algorithm to determine the angle of flap rotation from the induced astigmatism on the assumption that the free flap acts as a plus cylinder, while the stromal bed acts as a minus cylinder [1]. Na et al. [8] reported the successful repositioning of free flap according to the algorithm suggested by Hovanesian and Maloney [1]. However, all patients reported by Hovanesian and Maloney [1] as well as by $\mathrm{Na}$ et al. [8] presented relatively regular bow-tie astigmatism in topography in spite of misaligned cap, which would be favorable for rotation-angle calculation and good results after reposition surgery. Utz et al. [3] reported that irregular astigmatism caused by the intraoperative incidence of a free cap was corrected by repetitive flap rotation and customized photorefractive keratectomy [3].

In our case, the refractive error was caused by the free flap's misalignment due to the lack of preoperative corneal markings. Flap rotation and customized photorefractive keratectomy were considered to correct the refractive error. However, the change in the refractive index after flap rotation was unpredictable. Thus, flap rotation was performed based on the sagittal curvature of Scheimpflug topography. This method effectively improved the refractive error.

Although the introduction of IntraLase femtosecond laser has reduced the occurrence of LASIK flap complications [9], surgeons should pay attention to prevent free flap formation and loss of orientation of the cap because both patients and surgeon may experience challenged course to correct the irregular astigmatism. With a misaligned free flap, the most efficacious management procedure should be determined, and the sagittal curvature of Scheimpflug topography was a viable reference index for free flap repositioning in cases with inadequate preoperative markings.

\section{Statement of Ethics}

Written informed consent was obtained from the patient for publication of this case report and any accompanying images. In accordance with local guidelines, the need for ethical approval was not required by the Inje University's IRB Committee. All procedures followed were in accordance with the ethical standards and with the Helsinki Declaration.

\section{Conflict of Interest Statement}

We have no conflicts of interest to declare.

\section{Funding Sources}

This research received no specific grant from any funding agency in the public, commercial, or not-for-profit sectors.

\section{Author Contributions}

H.E.S. and H.J.H. made substantial contributions to the conception or design of the work. S.H.B. and S.Y.K. contributed to acquisition of data for the work. M.J.K., S.W.L, and H.J.H. drafted the work. H.E.S. and M.J.K. contributed equally to this work and should be considered co-first authors.

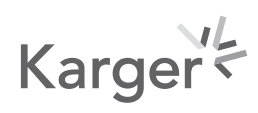




\section{Data Availability Statement}

All data generated or analyzed during this study are included in this article. Further enquiries can be directed to the corresponding author.

\section{References}

1 Hovanesian JA, Maloney RK. Treating astigmatism after a free laser in situ keratomileusis cap by rotating the cap. J Cataract Refract Surg. 2005 Oct;31(10):1870-6.

2 Todani A, Al-Arfaj K, Melki SA. Repositioning free laser in situ keratomileusis flaps. J Cataract Refract Surg. 2010 Feb;36(2):200-2.

3 Utz VM, Krueger RR. Management of irregular astigmatism following rotationally disoriented free cap after LASIK. J Refract Surg. 2008 Apr;24(4):383-91.

4 Stulting RD, Carr JD, Thompson KP, Waring GO 3rd, Wiley WM, Walker JG. Complications of laser in situ keratomileusis for the correction of myopia. Ophthalmology. 1999 Jan;106(1):13-20.

5 Fogla R, Padmanabhan P. Interrupted sutures at the hinge site to manage a free flap during laser in situ keratomileusis. J Cataract Refract Surg. 2004 Oct;30(10):2235-8.

6 Cheng AC, Wong VW, Rao SK, Lam DS. Repositioning of free cap four days after LASIK. J Refract Surg. 2007 June;23(6):625-7.

7 McLeod SD, Holsclaw D, Lee S. Refractive, topographic, and visual effects of flap amputation following laser in situ keratomileusis. Arch Ophthalmol. 2002 Sep;120(9):1213-7.

$8 \mathrm{Na}$ M, Chung SK, Pak KH, Choi JS. Repositioning of a free cap after laser-assistedin situKeratomileusis. J Korean Ophthalmol Soc. 2018 May;59(5):484-90.

9 Hashmani S, Hashmani N, Rajani H, Ramesh P, Soomro JA, Hussain Shah SR, et al. Comparison of visual acuity, refractive outcomes, and satisfaction between LASIK performed with a microkeratome and a femto laser. Clin Ophthalmol. 2017 May;11:1009-14. 